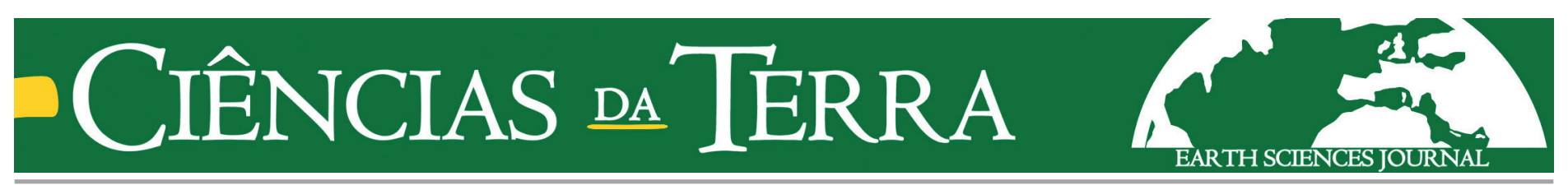

Check for updates

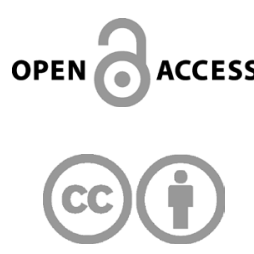

\section{António Nascimento Joaquim (1933 - 2013)}

\author{
M. T. Antunes ${ }^{1,2}$, R. B. da Rocha ${ }^{1} \&$ J. C. Kullberg ${ }^{1}$ \\ ${ }^{1}$ GeoBioTec, Earth Sciences Department, Faculty of Sciences and Technology, Universidade NOVA Lisboa, \\ Campus de Caparica.2829-516 Caparica, Portugal.rbr@fct.unl.pt; jck@fct.unl.pt \\ ${ }^{2}$ Academia das Ciências de Lisboa, R. da Academia das Ciências, 1249-122 Lisboa, Portugal. \\ migueltellesantunes@gmail.com
}

Corresponding author:

R. B. Rocha

rbr@fct.unl.pt

\section{Journal webpage:}

http://cienciasdaterra.novaidfct.pt/index. php/ct-esj/article/view/365

\section{Copyright:}

(C) 2017 M.T. Antunes, R. B. Rocha \& J. C. Kullberg. This is an open access article distributed under the terms and conditions of the Creative Commons Attribution License (CC BY), which permits unrestricted use, distribution, and reproduction in any medium, provided the original author and source are credited.
Resumo - Iniciou a sua carreira profissional como Naturalista no Museu e Laboratório Mineralógico e Geológico da FC/UL e terminou-a como Investigador Principal da FCT/ UNL, depois de se ter doutora-do em Geologia (Estratigrafia e Paleontologia) nesta Universidade. Dedicou-se ao estudo das associa-ções de ostracodos do Terciário português, tendo inventariado mais de 150 espécies (algumas novas) assinaladas pela primeira vez em Portugal.

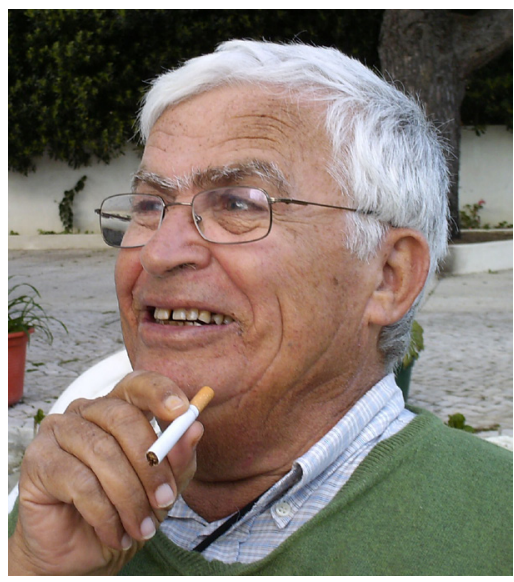

Nasceu em Afonso Vicente (Alcoutim) a 11 de Junho de 1933 e faleceu em Cascais a 20 de Dezembro de 2013.

Frequentou na Faculdade de Letras da Universidade de Lisboa o Curso de Professores Adjuntos do $11^{\circ}$ Grupo do Ensino Técnico Profissional, concluído em 1966. Com esta habilitação, exerceu funções de professor do Ensino Secundário Oficial de 3 de Outubro de 1966 a 30 de Junho de 1971.

Durante este período de 4 anos frequentou na Faculdade de Ciências de Lisboa a licenciatura em Ciências Geológicas, que concluiu em Maio de 1970 com classificação atingindo 14 valores por beneficiar da nota de estágio (18 valores) que um de nós (R.R.) lhe atribuiu pelo trabalho de campo no Algarve.

Em Junho de 1971 foi nomeado Técnico Experimentador da Faculdade de Ciências da Universidade de Lisboa e contratado, em Julho de 1973, como Naturalista do quadro do Museu e Laboratório Mineralógico e Geológico da mesma Faculdade, cargo que exerceu até Outubro de 1979.

Embora autorizada, a sua contratação como Investigador além do quadro daquele Museu não chegou a ser efetuada, por entretanto ter preferido ser transferido, mediante intervenção nossa, para a
Universidade Nova de Lisboa, já que havia começado a trabalhar connosco (MTA). Com efeito, em 1973 propusemos-lhe o estudo dos ostracodos do Neogénico de Portugal como tema de trabalho. Aceitou. Ninguém da FCL tentou retê-lo.

Assim, em Novembro de 1979 ingressou como Técnico Investigador da Faculdade de Ciências e Tecnologia da UNL, tendo sido reclassificado, um mês depois, como Investigador Auxiliar.

Durante a sua carreira na Universidade Nova de Lisboa participou no Senado da UNL, no Conselho da FCT, no Conselho Científico da FCT, na Assembleia de Representantes e no Conselho do Departamento de Ciências da Terra.

Foi promovido a Investigador Principal no final da década de noventa, após processo de aprovação muito difícil, que acabou por passar devido à nossa (MTA) insistência junto do Conselho Científico da Faculdade, invocando a esperança de que elaborasse uma síntese sobre os ostracodos do Neogénico, o que não fez.

\section{Investigação}

Iniciou a carreira de investigação no Centro de Estudos de Geologia Pura e Aplicada da Faculdade 
de Ciências da Universidade de Lisboa (1974-1975) onde integrou o projeto "Estudos geológicos do Alentejo, do Algarve e da Orla Ocidental", patrocinado pelo Instituto de Alta Cultura (IAC) e dirigido pelo Professor Carlos Teixeira.

Entre 1970 e 1973 participou em trabalhos de cartografia geológica no Algarve (carta de Lagos) e Alentejo (formações pré-câmbricas e paleozóicas do Alto Alentejo, maciço ígneo de Ervedal). Um dos principais responsáveis directos destes trabalhos, foi o nosso Colega Francisco Álvaro Gonçalves.

Por outro lado, revelava elevada capacidade em termos de paciência, o que parecia apropriado para se ocupar de um tema até então não desenvolvido em Portugal, o estudo dos ostracodos do Neogénico, desde logo admitido como tema de Doutoramento a preparar sob nossa (MTA) orientação.

A partir do final de 1973, e com a anuência das entidades diretivas da Faculdade de Ciências, onde ainda se mantinha, passou a dedicar-se aos ostracodos cenozóicos das bacias do Tejo e do Algarve, integrando a equipa de nossa (MTA) responsabilidade na linha de ação designada, primeiro, por "Geologia e Paleontologia de formações meso-cenozóicas" e, mais tarde, "Estudo das bacias cenozóicas portuguesas".

Em 1975 ingressou no Centro de Estratigrafia e Paleobiologia (CEPUNL) da UNL, patrocinado pelo Instituto Nacional de Investigação Científica e, mais tarde, pela Junta Nacional de Investigação Cientifica e Tecnológica. Após a reorganização do CEPUNL, em 1994, passou a integrar a linha de investigação "Estudos estratigráficos, paleontológicos e hidrogeológicos" do Centro de Estudos Geológicos (CEG), financiado pela Fundação para a Ciência e a Tecnologia (1994-2001).

Com apoio de M.T.A., não lhe faltaram meios. Foram-lhe proporcionados, mediante bolsas de estudo concedidas pelo IAC (1974), pelo Governo Francês $(1977,1978,1980$ e 1983) e pelo INIC (1981 e 1986), estágios em laboratórios adequados, permitindo comparar ostracodos portugueses com espécimes de outras proveniências:

- Centre de Paléontologie Stratigraphiquel Université Claude Bernard, Lyon 1 (França) com Gilles Carbonnel;

- Institut de Géologie du Bassin d'Aquitaine (Pau, França) e Centre de Recherche sur les Environnements Sédimentaires et Océaniques da Université de Bordeaux I, com Odette Ducasse, Pierre Carbonel e Bruno Cahuzac;
- Département de Production de la Société ELF-Aquitaine (Pau, França) com Henri J. Oertli;

- Istituto e Museo di Geologia dell'Università di Palermo (Itália) com Giuliano Ruggieri;

- Stazione Zoologica da Università di Napoli (Itália) com Gioacchino Bonaduce;

- Moravian Museum de Brno (República Checa) com Jaroslav Riha.

Prosseguiu, apesar do contratempo que constituiu o incêndio da Faculdade de Ciências de Lisboa, em 18 de Março de 1978; perderam-se documentos e amostras.

Participou em reuniões (Belgrado, 1979; Houston, 1982; Frankfurt, 1989; Barcelona, 1990; Warrnanbool, Austrália, 1991; Lisboa, 1992; Glasgow, 1993; Tours, 1995). Contactos com ostracodologistas ajudaram-no a inventariar 158 espécies, todas assinaladas pela primeira vez em Portugal. Foram descritas e consideradas novas as seguintes:

Pokornyella lisitanica Nascimento, 1978

Loxoconcha tagana Nascimento, 1980

Aurila zbyszewskii Nascimento, 1984

Cytheretta (C.) ferreirae Nascimento, 1987

Cytheretta (C.) ramalhi Nascimento, 1988

Cytherella (C.) antunesi Nascimento, 1988

Cytherella (C.) moyesi Nascimento, 1988

Neonesidae rochae Nascimento, 1988

Callistocythere oertlii Nascimento, 1988

Cyamocytheridea carbonneli Nascimento, 1988

Aurila (Cimbaurila) peypouqueti Nascimento, 1988

Hermanites ruggierii Nascimento, 1988

Loxoconcha (L.) carboneli Nascimento, 1988

Loxoconcha (L.) ducasseae Nascimento, 1988

Loxoconcha (Sagmatocythere) bonaducea Nascimento, 1988

Xestoleberis (X.) paisi Nascimento, 1988.

O Doutoramento em Geologia, especialidade de Estratigrafia e Paleontologia, foi concretizado na Faculdade de Ciências e Tecnologia da UNL em 1988, 15 anos após o começo. A tese foi intitulada "Ostracodos do Miocénico da Bacia do Tejo: sistemática, biostratigrafia, paleoecologia, paleogeografia e relações Mediterrâneo-Atlântico". O contratempo da perda do material que ardeu em 1978 no Museu Mineralógico e Geológico da Faculdade de Ciências e, sobretudo, a natural morosidade, explicam a demora: ia-nos (MTA) dizendo "que estava quase pronto"... durante quatro anos!

Do seu Curriculum constam 40 trabalhos publicados entre 1972 e 1999. 
Foi-lhe dedicada a espécie Leptacodon nascimentoi Estravís, 1992, micromamífero do Eocénico Inferior de Silveirinha (Baixo Mondego).

Foi membro da Sociedade Geológica de Portugal, da Associação Portuguesa de Geólogos, da Liga de Proteção da Natureza, da Sociedade Portuguesa de Ciências Naturais (membro da Direção em 19801981) e do Grupo de Ostracodologistas de Língua Francesa. Foi delegado para Portugal da European Ostracod Association e da revista "Cypris - International Ostracoda News Letter".

O Nascimento foi um bom colega, "bonacheirão", moderadamente ativo entre cigarradas, pai extremamente dedicado, bom companheiro em trabalhos de campo, ostracodologista minucioso, sério no seu trabalho. Revelava paixão pelas suas pesquisas, de que se ufanava, ao ponto de inflacionar hipotéticos resultados.

Entre os objetivos que ele próprio apontou para a prossecução de investigação pós-doutoral, contavam-se:

- a elaboração de uma síntese paleoecológica acerca dos ambientes marinhos (infralitoral, circalitoral), salobros e de água doce do Neogénico português, com base em associações de ostracodos, que não realizou;

- o assinalar, pela primeira vez, a presença de ostracodos glaciários no Minho;

- a caracterização paleontológica do Oligocénico marinho da Bacia do Tejo e de sedimentos aquitanianos na Bacia do Sado; porém, estas interpretações são incompatíveis com melhores dados, já então conhecidos e não se confirmam;

- estabelecer uma escala de biozonação para o Miocénico português com base em associações de ostracodos, não realizada.

Os resultados ficam aquém dos objetivos. Deixou uma lista das espécies identificadas, o que é positivo mas limitado. Infelizmente, acabou por não tirar partido de uma das facetas mais instrutivas dos ostracodos, o seu significado ecológico, nem as ocorrências foram claramente integradas no contexto estratigráfico.

A situação obrigou outros (Meco, Legoinha \& Antunes, 2003) a trabalhar os seus dados para tentar tirar algum partido de todo o trabalho que lhe cabia ter feito, criando uma data base para o Neogénico de Portugal com informação acerca de 158 espécies de ostracodos provenientes de 16 cortes geológicos e duas sondagens.

Era seu desejo ser promovido a Investigador Principal. O processo era difícil por a atribuição de verbas reverter, com prioridade, para a Carreira Docente, agravado pela modéstia do Currículo. Decorreram alguns anos de diligências junto dos votantes, no sentido de conseguir a aprovação, invocando o muito que haveria a esperar do seu trabalho. Enfim, a proposta passou. Entregou, em 2001, os papéis para a reforma.

Ficam a recordação e a saudade de um colega bem-humorado e sempre disponível para nos acompanhar.

\section{Anexo - Publicações (por ordem cronológica)}

Nascimento A., Ladeira F. \& Coelho A. V. P. (1972) - Contribuição para o conhecimento geológico do maciço eruptivo do Ervedal (Avis). Bol. Mus. Lab. Min. Geol. Fac. Ciências Lisboa 13 (1), 97-131.

Gonçalves F., Ladeira F. \& Nascimento A. (1973) - Carta Geológica de Portugal na escala de 1/50 000, Folha 32-D, Sousel. Serv. Geol. Portugal, Lisboa.

Chevalier J. P. \& Nascimento A. (1975) - Notes sur la Géologie et la Paléontologie du Miocène de Lisbonne. XVI - Contribution à la connaissance des madréporaires et des faciès récifaux du Miocène inférieur. Bol. Soc. Geol. Portugal XIX (III), 247-281. http://socgeol.org/documents/notes-sur-lageologie-et-la-paleontologie-du-miocene-de-lisbonne-xvicontribution-a-la-connaissance-des-madreporaires-et-desfacies-recifaux-du-miocene-inferieur

Canelhas M. G. S. \& Nascimento A. (1976) - O Museu e Laboratório Mineralógico e Geológico no contexto do Museu Nacional de História Natural. In Panorama Museológico Português, Carências e Potencialidades (Lisboa, 1979). Actas Colóquio APOM 76, 131-136.

Canelhas M. G. S. \& Nascimento A. (1976) - Algumas considerações sobre uma visita de estudo a Museus de História Natural dos Estados Unidos da América do Norte. Bol. Soc. Geol. Portugal XX, 317-348. http://socgeol.org/documents/ algumas-consideracoes-sobre-uma-visita-de-estudo-a-museus-de-historia-natural-dos-estados-unidos-da-americado-norte

Nascimento A. (1978) - Notes sur la Géologie et la Paléontologie du Miocène de Lisbonne. XXII - Une espèce nouvelle, Pokornyella lusitanica (Ostracoda), dans le Miocène inférieur du Bassin du Tage. Ciências da Terra 4, 47-58. http://cienciasdaterra.novaidfct.pt/index.php/ct-esj/article/view/55

Nascimento A. (1980/81) - Notes sur la Géologie et la Paléontologie du Miocène de Lisbonne. XXIII - Loxoconcha tagana (Ostracoda), nouvelle espèce du Miocène inférieur de Lisbonne. Bol. Soc. Geol. Portugal XXII, 33-35. http://socgeol. org/documents/notes-sur-la-geologie-et-la-paleontologiedu-miocene-de-lisbonne-xxii-loxoconcha-tagana-ostracoda-nouvelle-espece-du-miocene-inferieur-de-lisbonne

Teixeira C., Gonçalves F., Nascimento A. \& Fernandes S. C. (1981) - O maciço eruptivo da Serra de Sintra. SPCN Col. Natura 6, 1-48. 
Antunes M. T., Jonet S. \& Nascimento A. (1981) - Vertébrés (Crocodiliens, poissons) du Miocène marin de l'Algarve occidentale. Ciências da Terra 6, 9-38. http://cienciasdaterra. novaidfct.pt/index.php/ct-esj/article/view/74

Antunes M. T., Bizon G., Nascimento A. \& Pais J. (1981) - Nouvelles données sur la datation des dépôts miocènes de l'Algarve (Portugal) et l'évolution géologique régionale. Ciências da Terra 6, 153-168. http://cienciasdaterra.novaidfct.pt/ index.php/ct-esj/article/view/79

Nascimento A. (1981) - Les ostracodes du Miocène inférieur de la région de Lisbonne /Bassin du Tage). Comum. VII Intern Symp. Ostracods, Belgrade, 1979. Ciências da Terra 6, 189-196. http://cienciasdaterra.novaidfct.pt/index.php/ctesj/article/view/81

Antunes M. T. (col. de Baptista A. P., Bravo M. S., Cappetta F. C., Coelho A. V. P., Costa A. T. M., Ferreira O. V., Furtado A. S., Gomes F., Mein P., Nascimento A., Pais J., Rocha R. B. \& Oliveira J. T.) (1983) - Carta Geológica de Portugal na escala de 1/50 000. Notícia explicativa da Folha 39-C, Alcácer do Sal. Serv. Geol. Portugal, Lisboa.

Nascimento A. (1983) - The Ostracod fauna of the Portuguese Neogene and its relationship to those from the Atlantic and Mediterranean basins. In: Maddocks R. F., Applications of Ostracoda. Proc. VIII Intern. Symp. Ostracods, Houston, Geosciences, 429-436.

Nascimento A. (1984) - Aurila zbyszewskii (Ostracoda) espèce nouvelle du Miocène supérieur du Portugal. Ed. Recherches sur les civilisations, Vol. d'hommage au Géologue G. Zbyszewski, Paris, 333-338.

Antunes M. T., Mein P., Nascimento A. \& Pais J. (1986) - Le gisement pleistocène de Morgadinho, en Algarve. Ciências da Terra 8, 9-22. http://cienciasdaterra.novaidfct.pt/index. $\mathrm{php} / \mathrm{ct}-\mathrm{esj} / \mathrm{article} / \mathrm{view} / 88$

Nascimento A. (1987) - Cytheretta (Cytheretta) ferreirae (Ostracoda) nova espécie do Miocénico inferior da Bacia do Tejo (Portugal). In: Da pré-história à história, Vol. homenagem a Octávio da Veiga Ferreira. Edit. Delta, 67-70.

Nascimento A. \& Silva P. (1987) - Ostracodos da plataforma continental portuguesa ao largo do rio Minho. Reunião aberta sobre geologia marinha, Lisboa 1988. Relatório Disepla $2 / 88$, p. 39.

Nascimento A. (1988) - Ostracodos do Miocénico da Bacia do Tejo: sistemática, biostratigrafia, paleoecologia, paleogeografia e relações Mediterrâneo - Atlântico. Tese Univ. Nova Lisboa (não publicada), $306 \mathrm{p}$.

Nascimento A. \& Silva P. (1989) - Ostracodos da plataforma norte minhota: testemunhos de ambientes actuais e quaternários. Semin. "A Geologia e o Ambiente”, Aveiro (comun. em painel).

Nascimento A. \& Silva P. (1989) - Primeira notícia sobre ostracodos glaciários na vertente continental superior do Minho. Gaia, Rev. Geociências, Mus. Nac. Hist. Nat., Lisboa, 1, 25-27.

Nascimento A. (1990) - Nouvelles espèces d'ostracodes du Miocène du Bassin du Tage (Portugal). Ciências da Terra 10,
107-126. http://cienciasdaterra.novaidfct.pt/index.php/ctesj/article/view/122

Nascimento A. (1990) - Tentative ostracode biozonation of the Portuguese Neogene. Cour. Forsch.-Inst. Senckenberg, Frankfurt, 123, 181-190.

Nascimento A. (1990) - Nouvelles données stratigraphiques et paléoécologiques sur le Miocène de la région de Lisbonne, basées sur l'étude des ostracodes. $6^{\text {th }}$ Meeting European Geol. Societies, Programme and Abstracts, Lisboa, p. 61.

Antunes M. T., Civis J., Gonzalez Delgado J., Gavilán G. A., Andrés I., Pais J., Sierro J. F., Valle M. \& Nascimento A. (1990) - The Late Serravallian-Early Tortonian marine sediments of the Tagus Basin (Lisbon region). A sedimentological and paleontological approach. IX Congress RCMNS, Barcelona, 27-29.

Martins J. M. B., Azevedo T. M., Nascimento A., Ubaldo M. L. \& Ramalho M. M. (1991) - O limite estratigráfico Oligocénico-Miocénico na região do Barreiro (Península de Setúbal) - Estudo micropaleontológico da sondagem S48/ Br1. III Congr. Nac. Geologia, Coimbra, p. 118.

Nascimento A. (1991) - Primeira notícia sobre terrenos de idade aquitaniana na Bacia do Sado. III Congr. Nac. Geologia, Coimbra, p. 120.

Nascimento A. \& Azevedo M. T. (1991) - Primeira caracterização paleontológica do Oligocénico marinho, na Bacia do Tejo (Portugal). III Congr. Nac. Geologia, Coimbra, p. 121.

Nascimento A. (1992) - Testemunhos megalíticos de Afonso Vicente (Alcoutim) - notícia preliminar. Almadan, Centro Arqueologia Almada, sér. II, 1, 92-93.

Lauriat-Rage A., Brébion P., Cahuzac B., Chaix C., Ducasse O., Ginsburg L., Janin M.-C., Lozouet P., Margerel J., Nascimento A., Pais J., Poignant A., Pouyet S. \& Roman J. (1993) - Palaeontological data about the climatic trends from Chattian to present along the Northeastern Atlantic frontage. Proceed. I Cong. RCANS, Lisboa 1992. Ciências da Terra 12, 167-179. http://cienciasdaterra.novaidfct.pt/index. php/ct-esj/article/view/160

Nascimento A. (1993) - Application of abundances of Cyprideis group taxa and marine species to the reconstitutions of Aquitanian paleoenvironments in the Tagus Basin (Portugal). In: Mckenzie K. G. \& Jones P. J. (Eds.), Ostracoda in the earth and life sciences. A. A. Balkema, Rotterdam, 229-240.

Nascimento A. \& Riha J. (1993) - Ostracod species common to the Neogene of Portugal and Central Paratethys. 2nd Europen Ostracodologists Meet., Glasgow, 169-176.

Antunes M. T., Legoinha P., Nascimento A. \& Pais J. (1995) Biostratigrafia e paleoambientes do Miocénico do flanco sul do sinclinal de Albufeira (Península de Setúbal). Mem. Museu Lab. Miner. Geol. Fac. Ciênc. Porto 4, 19-23.

Antunes M. T., Legoinha P., Pais J. \& Nascimento A. (1995) Evolução da Bacia do Baixo Tejo (Lisboa e Península de Setúbal, Portugal) no Burdigaliano e Langhiano. Mem. Museu Lab. Miner. Geol. Fac. Ciênc. Porto 4, 23-28. 
Carbonel P., Ducasse O. \& Nascimento A. (1995) - Ruggieria (Ruggieria) micheliana (Ostracoda), marqueur d'un changement climatique entre Aquitanien et Burdigalien dans le Bassin du Tage. Interim-colloquium "Atlantic Neogene climates and paleoenvironments", Tours, p. 12.

Antunes M. T., Legoinha P., Nascimento A. \& Pais J. (1996) - The evolution of the Lower Tagus basin (Lisbon and Setúbal peninsula, Portugal) from Lower to earlier Middle Miocene. Géol. France, Orléans, 4, 59-77.

Antunes M. T., Civis J., González-Delgado J. A., Legoinha P., Nascimento A. \& Pais J. (1996) - Miocene stable isotopes $\left(\delta^{18} \mathrm{O}, \delta{ }^{13} \mathrm{C}\right)$, biostratigraphy and environments in the southern limb of Albufeira syncline (Setúbal Peninsula, Portugal). Geogaceta 21, 21-24. http://www.sociedadgeologica. es/archivos/geogacetas/Geo21/Art05.pdf

Antunes M. T., Civis J., González-Delgado J. A., Legoinha P., Nascimento A. \& Pais J. (1997) - Lower Miocene stable isotopes $\left(\Delta 18^{\mathrm{O},} \Delta 13^{\mathrm{C}}\right)$, biostratigraphy and environments in the Foz da Fonte and Penedo sections (Setúbal Peninsula,
Portugal). Geogaceta 23, 7-10. http://www.sociedadgeologica.es/archivos/geogacetas/Geo23/Art02.pdf

Antunes M. T., Elderfield H., Legoinha P., Nascimento A. \& Pais J. (1998) - Sequências deposicionais, biostratigrafia e idades isotópicas do Miocénico da bacia do Baixo Tejo (Lisboa, Península de Setúbal, Portugal). Com. Inst. Geol. Min. 84 (1), A134-A137.

Antunes M. T., Elderfield H., Legoinha P., Nascimento A. \& Pais J. (1999) - A stratigraphic framework for the Miocene from the Lower Tagus Basin (Lisbon, Setúbal Peninsula, Portugal). Depositional sequences, biostratigraphy and isotopic ages. Bol. Soc. Geol. España 12 (1), 3-15. http://www.sociedadgeologica.es/archivos/REV/12(1)/Art01.pdf

Antunes M. T., Carbonel P., Colin J.-P. \& Nascimento A. (1999) - On the Upper Cretaceous age and affinities of Neocyprideis lusitanicus (Ostracoda). Ciências da Terra 13, 87- 92. http://cienciasdaterra.novaidfct.pt/index.php/ct-esj/article/ view/169 